I am grateful to the Council for Scientific and Industrial Research and to Prof. Immelman and Mr. Brits, of the Department of Physics, for technical aid.

University of the Orange Free State, G. Erofr

Bloemfontein.

Sept. 14

${ }^{1}$ Brit. J. Psych., 42, Pts. 1 and 2 (1951).

\title{
A Quantum Mechanical Study of Lithium Hydride
}

THE electronic energy of lithium hydride has been computed by the Hartree-Fock approximation. The computations were carried out both by the linear combination of atomic orbitals approximation according to the molecular orbital method, and by the electron-pair approximation according to Heitler and London with inclusion of ionic terms. When only the two valence electrons were considered, the computed values of the binding energy were as follows:

Linear combination of atomic orbitals approximation of molecular orbitals

Electron-pair method without ionic terms

Electron-pair method with ionic term

Experimental value

$3.57 \mathrm{eV}$.

$2 \cdot 64$,

$2 \cdot 54 \pm 0.23 \mathrm{eV}$

In these computations the value of the interatomic distance, $R$, was put equal to the experimental value, $1 \cdot 6 \mathrm{~A}$. As the computed energy values are larger than the experimental value, the neglect of the repulsion between the valence electrons and the electrons in the inner shell of the lithium atom seems to cause a considerable error. This fact is in agreement with the result of James's calculations ${ }^{2}$ on the lithium molecule $\left(\mathrm{Li}_{2}\right)$. Therefore the energy of the lithium hydride molecule was computed with a wave function including molecular orbitals for all the four electrons of the molecule. This more accurate treatment resulted in a computed value of the binding energy of $2.0 \mathrm{eV}$. when $R=1.6 \mathrm{~A}$. The computations will be repeated with other values of this parameter. It seems reasonable to expect that these new computations will result in energy values not much different from the one computed already.

Although the value of the binding energy is evidently rather sensitive to the method of approximation, it may be hoped that the form obtained for the wave function and thus the electron distribution is more insensitive. Both in the two electron cases and in the four-electron case, the best values of the parameters in the wave function indicate that the structure $\mathrm{Li}^{+} \mathrm{H}^{-}$is the most important one. This fact emphasizes that no significance should be attributed to the apparently excellent agreement between the experimental value of the binding energy and that computed according to the electron-pair method without ionic terms. The same objection is applicable to the calculations on lithium hydride by Hutchisson and Muskat ${ }^{3}$. The calculated structure $\mathrm{Li}^{+} \mathrm{H}^{-}$is in good agreement with the experimental fact that hydrogen is obtained at the anode during electrolysis of lithium hydride.

Another interesting point in the results of the calculations is the relative importance of $2 s$ - and $2 p$-orbitals around the lithium atom, that is, the degree of 's,p-hybridization'. If this degree of hybridization is calculated according to the criterion of maximum overlapping, the $s$ - and $p$-orbitals are found to be of about equal importance. Almost the same result is obtained when only the two valence electrons are considered in the calculations of the energy. However, when the molecular orbitals of all the four electrons are included in the wave function, the $2 p$-orbital is found to be much less important than the $2 s$-orbital. This result seems to be reasonable, as the $2 p$-energy-level of the free lithium atom lies as much as $1.85 \mathrm{eV}$. above the ground-level.

Full details of this investigation will be published elsewhere.

Institute of Theoretical Physics,

INGA FISCHER

University of Stockholm. June 23.

${ }^{1}$ Crawford, F. H, and Jorgensen, jun, T, Phys, Rev, 49, 745 (1936). ${ }^{2}$ James, H. M., J. Chem. Phys., 2, 794 (1934).

Hutchisson, E., and Muskat, M., Phys. Rev., 40, 340 (1932).

\section{Demonstration of Bimolecular Anionotropic Change}

THovgH it has long been accepted that the unimolecular process $S_{N} \mathbf{l}^{\prime}$ represents a common mechanism of isomeric rearrangement and of substitution with rearrangement in anionotropic systems, for example,

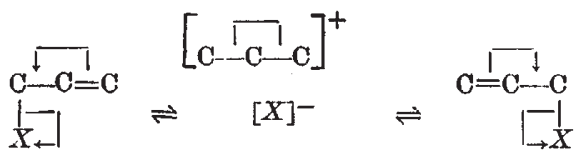

the idea has been extant since $1938^{1}$ that a bimolecular mode of rearrangement $S_{N} 2^{\prime}$ might also exist :

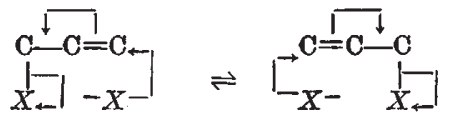

In these formulations $X^{-}$represents a general anion, so that the $X$ 's which engage in different processes, or together participate in the same process, may, or may not, be chemically identical.

The earlier attempts ${ }^{2}$ to detect mechanism $S_{N} 2^{\prime}$ resulted $(a)$ in producing new evidence substantiating the mechanism $S_{N} l^{\prime}$, and $(b)$ in showing that, under conditions leading only to bimolecular reactions, any bimolecular substitution with rearrangement $S_{N} 2^{\prime}$ was so relatively unimportant as to be masked by bimolecular substitution without rearrangement $S_{N} 2$ in the systems studied.

There is, however, one group of cases in which evidence for bimolecular rearrangement $S_{N} 2^{\prime}$ has been claimed. They are all of one type : the entering substituent comes from a pseudo-acid. By treatment with ethyl acetoacetate and a basic catalyst, cinnamyl alcohol has been converted, probably through its esters, to 1-phenylallylacetone, and 1-phenylallyl alcohol has been similarly converted to cinnamyl. aceton $\mathrm{e}^{3}$ :

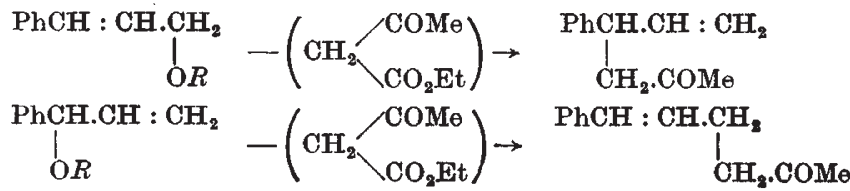

